\title{
INFLUENCE OF NUMERICAL SOIL MODEL TYPE ON DYNAMIC RESPONSE OF REINFORCED CONCRETE FRAME STRUCTURES
}

Scientific paper

\section{Patricija Ravlić}

(Received: 5 June 2020; accepted: 23 September 2020)

Josip Juraj Strossmayer University of Osijek, Faculty of Civil Engineering and Architecture Osijek, mag. ing. aedif.

\section{Ivan Kraus}

Josip Juraj Strossmayer University of Osijek, Faculty of Civil Engineering and Architecture Osijek, Dr.Sc, Assistant Professor

Corresponding author: ikraus@gfos.hr

\begin{abstract}
In engineering practice, numerical models of structures are typically supported using nondeformable media. However, the compliance and stratification of the underlying soil influence the seismic response of the structure. Its influence on structures subjected to dynamic loads has been investigated using various approaches to soil foundation modeling. This study was conducted for 70 different cases. Three actual soil profiles and two structures were analyzed, and the soil was modeled in three ways. Dynamic analysis was performed using five records of previous earthquakes. Finally, the relationship between the magnitude of the top displacement of the structure and the frequency content of ground motions was established.
\end{abstract}

Keywords: Foundation soil; reinforced concrete frame structure; ground motion records; predominant excitation period; mean excitation period; soil stratification. 


\section{INTRODUCTION}

In engineering practice, it is common to support numerical models of structures using nondeformable media. The compliance and stratification of the underlying soil affect the seismic response of a structure, and the soil-structure interaction (SSI) influences the dynamic response of structures supported by flexible soils ([1] and [2]). When engineers analyze a structure, they usually assume that the structure is fixed at its base, which is acceptable if the structure is supported on a rock. However, if the structure is constructed on compliant soil, the oscillation period and system damping will increase [3]. Maher, Osama, and Mohamed [4] investigated the response of reinforced concrete buildings subjected to various ground motion excitations considering the SSI and using PLAXIS. Kraus and Džakić [5] analyzed different approaches to soil modeling for numerical models and compared the behavior of conventional fixed structures with structures placed on Winkler springs and half-spaces. A linear analysis was performed on three different reinforced concrete frames using a time-history analysis and SAP2000. Khalii, Sadek, and Shahrour [6] evaluated the effect of SSI on the period of oscillation of a structure. The behaviors of the soil and the structure were assumed to be elastic, while the soil flexibility was modeled using springs. The effect of SSI was observed for single-story and multi-story models with one or more spans. In the studies cited in this section, the frequency content of ground motions was not investigated, and the dynamic responses of structures were ignored. Moreover, the ground motions applied in the studies were primarily selected and observed with respect to the maximum acceleration.

In this study, 70 numerical models were developed to investigate the dynamic behavior of frame structures supported by a deformable medium. Two reinforced concrete frames extracted from actual buildings with different geometries were modeled on three actual soil profiles and subjected to five actual ground motions. The observed structures were analyzed based on different base conditions. A fixed-base condition was adopted for a referent model. The results obtained using these models were compared to those for structures resting on a flexible medium represented by springs and dashpots and two-dimensional (2D) finite elements forming a half-space. The influence of soil stratification on the behavior of structures subjected to dynamic loading was also investigated. Finally, the relationship between the magnitude of the top displacement of the structure and the frequency content of ground motions was established.

\section{RESEARCH METHODOLOGY}

SSI effects are explored using the same soil-structure system modeled using three methods: a) the structure is fixed at its base, $b$ ) the structure is placed on a set of elastic springs and dashpots, and $c$ ) the structure is placed on 2D thick-shell finite elements forming a half-space (Figure 1).
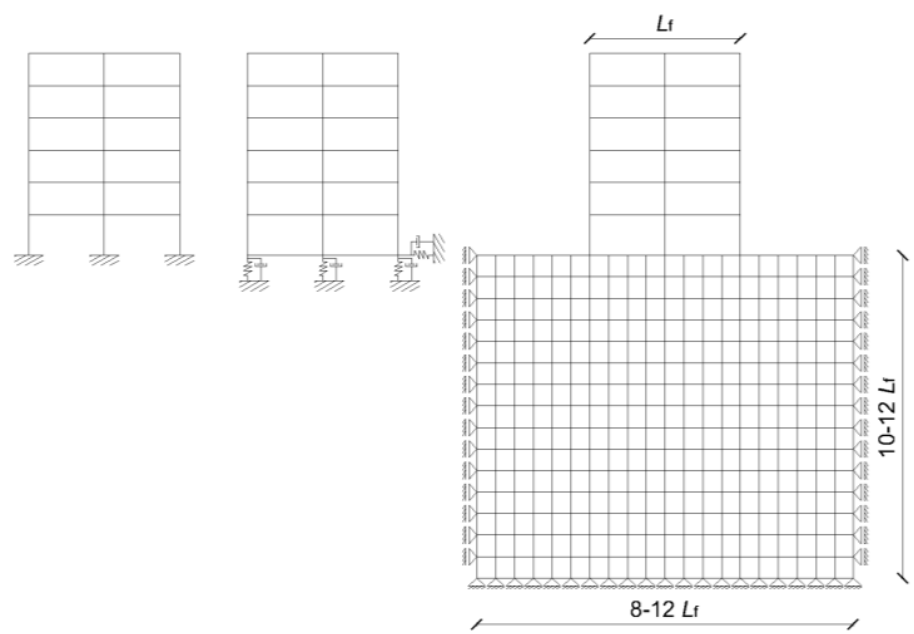

Figure 1 Schematic of soil-structure systems [7]: fixed support (left), springs and dashpots (middle), and half-space of 2D finite elements (right)

Ravlić, P, Kraus, I 


\subsection{Springs and dashpots}

When simulating the behavior of an actual structure using a fixed-base model, soil with infinite stiffness is assumed. Consequently, the calculation is directly made assuming that the foundation soil corresponds to Category A soil (i.e., rock according to [8]), regardless of the actual affiliation with possibly another category. On the contrary, actual structural support implies the flexibility (i.e., deformability) and interaction of integral components: the base elements and the soil. The deformability of the soil subjected to ground motion excitation can be simulated using springs and dashpots. In this study, the stiffness and damping were determined according to Pais and Kausel, as expressed in [7]:

$$
\begin{aligned}
& k_{\mathrm{x}, \text { total }}=\alpha_{\mathrm{x}} \times K_{\mathrm{x}, \text { sur }} \times \eta_{\mathrm{x}} \\
& k_{\mathrm{z}, \text { total }}=\alpha_{\mathrm{z}} \times K_{\mathrm{z}, \text { sur }} \times \eta_{\mathrm{z}} \\
& c_{\mathrm{x}, \text { total }}=2 \times k_{\mathrm{x}, \text { total }} \times\left(\frac{\beta_{\mathrm{emb}, \mathrm{x}} \times \beta_{\mathrm{sur}, \mathrm{x}}}{\omega}\right) \\
& c_{\mathrm{z}, \text { total }}=2 \times k_{\mathrm{z} \text { total }} \times\left(\frac{\beta_{\mathrm{emb}, \mathrm{z}} \times \beta_{\text {sur }, \mathrm{z}}}{\omega}\right)
\end{aligned}
$$

where $k_{x, \text { total }}$ is the total translational spring stiffness along the $x$-axis, $k_{z, \text { total }}$ is the total translational spring stiffness along the $z$-axis, $c_{x, \text { total }}$ and $c_{z, \text { total }}$ are the total translational dashpot coefficients along the $x$-axis and $z$-axis, respectively, $\omega$ is the undamped circular frequency, $\beta_{\mathrm{emb}, \mathrm{x}}$ and $\beta_{\mathrm{emb}, \mathrm{z}}$ are the radiation damping ratios for embedded footings along the $x$-axis and $z$-axis, respectively, and $\beta_{\text {sur, } x}$ and $\beta_{\text {sur }, z}$ are the radiation damping ratios for surface footings along the $x$-axis and $z$-axis, respectively. The radiation damping ratios were calculated as follows [7]:

$$
\begin{aligned}
& \beta_{\text {sur }, x}=\left[\frac{(4 \times(L / B))}{\left(K_{x, \text { sur }} /(G \times B)\right)}\right] \times\left[\frac{a_{0}}{2 \times \alpha_{x}}\right] \\
& \beta_{\text {sur }, z}=\left[\frac{(4 \times \psi \times(L / B))}{\left(K_{z, \text { sur }} /(G \times B)\right)}\right] \times\left[\frac{a_{0}}{2 \times \alpha_{z}}\right] \\
& \beta_{e m b, x}=\left[\frac{(4 \times[(L / B)+((D / B)(\psi+(L / B)))])}{\left(K_{x, \text { emb }} /(G \times B)\right)}\right] \times\left[\frac{a_{0}}{2 \times \alpha_{x}}\right] \\
& \beta_{\text {emb }, z}=\left[\frac{(4 \times[(L / B)+((D / B)(1+(L / B)))])}{\left(K_{z, \text { emb }} /(G \times B)\right)}\right] \times\left[\frac{a_{0}}{2 \times \alpha_{z}}\right]
\end{aligned}
$$

where $L$ and $B$ are the half-length and half-width of the entire foundation plan, respectively, $K_{\mathrm{x} \text {,sur }}$ and $K_{z \text { sur }}$ are static foundation stiffnesses of a rigid rectangular footing at the ground surface in the $x$-axis and $z$-axis, respectively, $G$ is the soil shear modulus at small strains, $a_{0}$ is the dimensionless frequency, and $\alpha_{x}$ is the dynamic stiffness modifier for rigid footings along the $x$-axis. $\psi$ is the soil-to-foundation stiffness ratio, which represented the flexibility of the foundation, $\alpha_{z}$ is the dynamic stiffness modifier for rigid footings along the $z$-axis, $D$ is the embedment depth, and $K_{\mathrm{x}, \text { emb }}$ and $K_{z, \text { emb }}$ are the static foundation stiffnesses of an embedded rigid rectangular footing in the $\mathrm{x}$-axis and $z$ axis, respectively. It should be noted that radiation damping refers to the radiation of seismic energy away from the foundation [7]. The dynamic stiffness modifiers and static foundation stiffness values for a rigid rectangular footing at the ground surface are calculated.

$$
\begin{aligned}
& \alpha_{x}=1.00 \\
& \alpha_{z}=1-\left[\frac{\left(0.4+\frac{0,2}{L / B}\right) \times a_{0}^{2}}{\left(\frac{10}{1+3 \times\left(L / B^{-1}\right)}\right)+a_{0}^{2}}\right] \\
& K_{x, \text { sur }}=\left(\frac{(G \times B)}{(2-v)}\right) \times\left[\left(6.8 \times(L / B)^{0,65}\right)+2.4\right] \\
& K_{z, \text { sur }}=\frac{G \times B}{1-v} \times\left[3.1 \times(L / B)^{0,75}+1,6\right]
\end{aligned}
$$

The soil-to-foundation stiffness ratio $\psi$ is obtained using the following equation [7]: 


$$
\psi=\min \left\{\sqrt{\frac{2(1-v)}{(1-2 v)}} ; 2.5\right.
$$

where $v$ is Poisson's ratio of soil, which is generally selected as 0.30 [7]. The dimensionless frequency $a_{0}$ is calculated as follows [7]:

$$
a_{0}=\frac{\omega \times B}{v_{s, 30}}
$$

where $\omega$ is the undamped circular frequency, and $v_{s, 30}$ is the average shear wave velocity in the upper $30 \mathrm{~m}$, which can be calculated as follows [8]:

$$
\mathrm{v}_{\mathrm{s}, 30}=\frac{30}{\sum \frac{\mathrm{h}_{\mathrm{s}, \mathrm{i}}}{\mathrm{v}_{\mathrm{s}, \mathrm{i}}}}
$$

where $h_{\mathrm{s} . \mathrm{i}}$ denotes the thickness $(\mathrm{m})$ of the $i$-th formation or layer, and $v_{\mathrm{s}, \mathrm{i}}$ denotes the shear-wave velocity of the ith layer in the top $30 \mathrm{~m}$.

\subsection{Half-space}

When the soil is modeled using 2D elastic finite elements, each soil layer can be modeled using different mass densities and moduli of elasticity [9]. In such a case, the soil model is supported on its entire bottom boundary by pin supports, while on its vertical boundaries, the soil model is restrained from a horizontal translation by roller-type supports. It is recommended that the vertical boundary of the soil model should be located at four to six widths of the foundation left and right from the center of the foundation, and the soil depth should be equal to or greater than 10 widths of the foundation [9]. The horizontal boundary of the soil model can be placed higher if the bedrock is located within a depth of 10 widths of the foundation. In addition, the vertical boundaries of the soil model can be placed three foundation widths from the foundation edge to minimize the influence of the boundary conditions [10].

\section{STRUCTURAL MODELS AND LOADS}

Two shallow-foundation reinforced concrete structures were modeled. One structure had two spans and six stories (further in text B2S6), and the other structure had six spans and seven stories (further in text B6S7). The structural elements were modeled using frame elements. The weight of the structural elements was automatically calculated by the computer program. A linear-elastic SSI was assumed, and the loads transferred from both structures to the ground were approximately equal. For each soil-structure system, a time-history analysis was performed using five actual ground motions. In total, 70 cases were analyzed (combinations of two structures, three soil profiles, three soil models, and five ground motions). The foundation depth was ignored, and the numerical modeling and analysis were conducted using SAP2000 [11].

\subsection{B2S6 structure [12]}

The elevation of the B2S6 structure and load distribution are shown in Figure 2. 


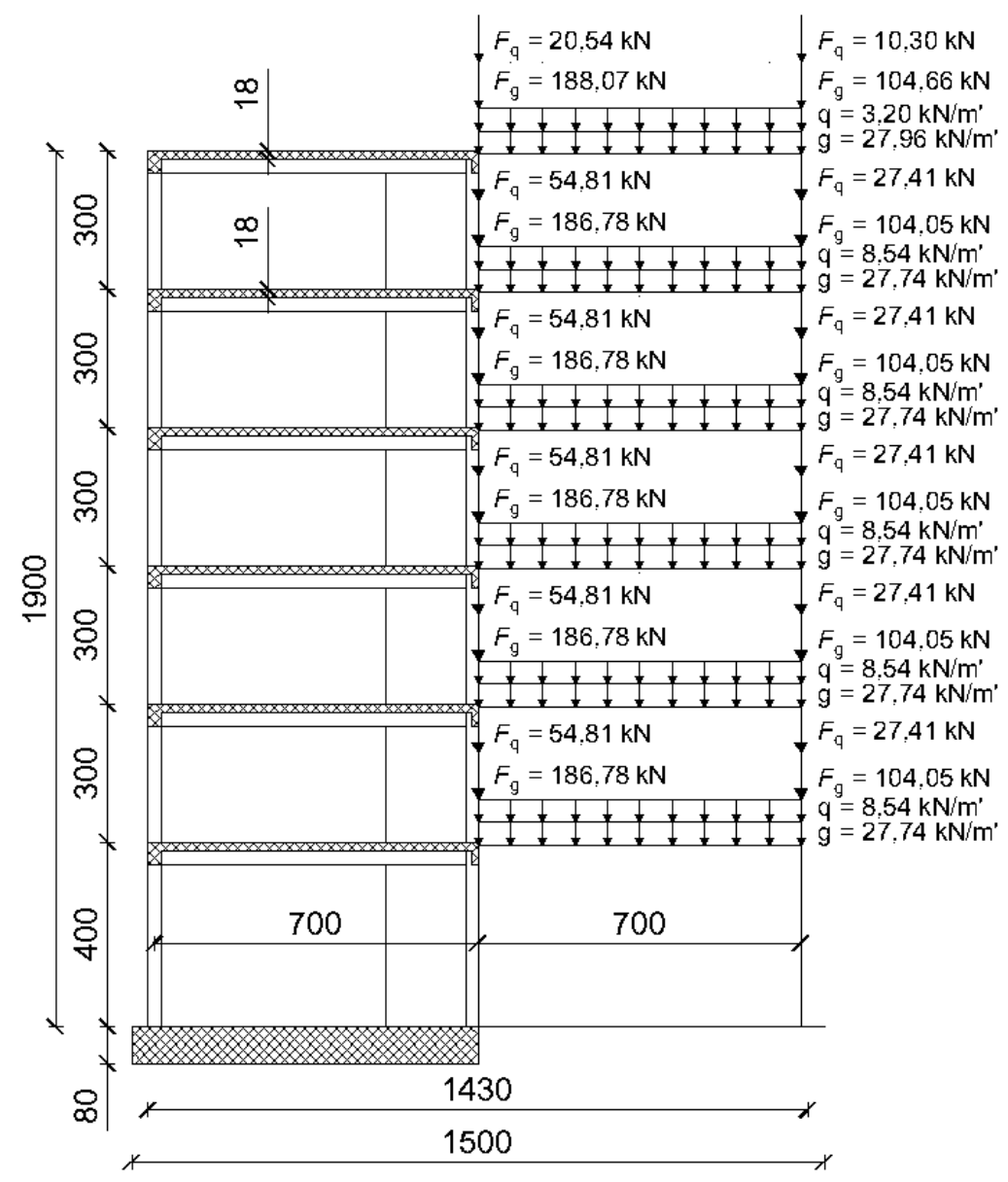

Figure 2 B2S6 structure ( $g$ is the dead load, and $q$ is the live load) [cm]

The height of the first story was $4 \mathrm{~m}$, and the height of the other stories was $3 \mathrm{~m}$ each. The clear length of all beams was $7 \mathrm{~m}$. Each external column had a rectangular cross-section of $30 / 70 \mathrm{~cm}$, each internal column had a cross-section of $50 / 50 \mathrm{~cm}$, the beams had a rectangular cross-section of $25 / 50 \mathrm{~cm}$ each, and the slabs were $18 \mathrm{~cm}$ thick. All elements were made of concrete class C25/30 and reinforcement S500 of class C. However, the reinforcement was not modeled in this study because it does not affect the behavior of linearly elastic systems.

\subsection{B6S7 structure [13]}

The elevation of the B6S7 structure and the load distribution are depicted in Figure 3. 


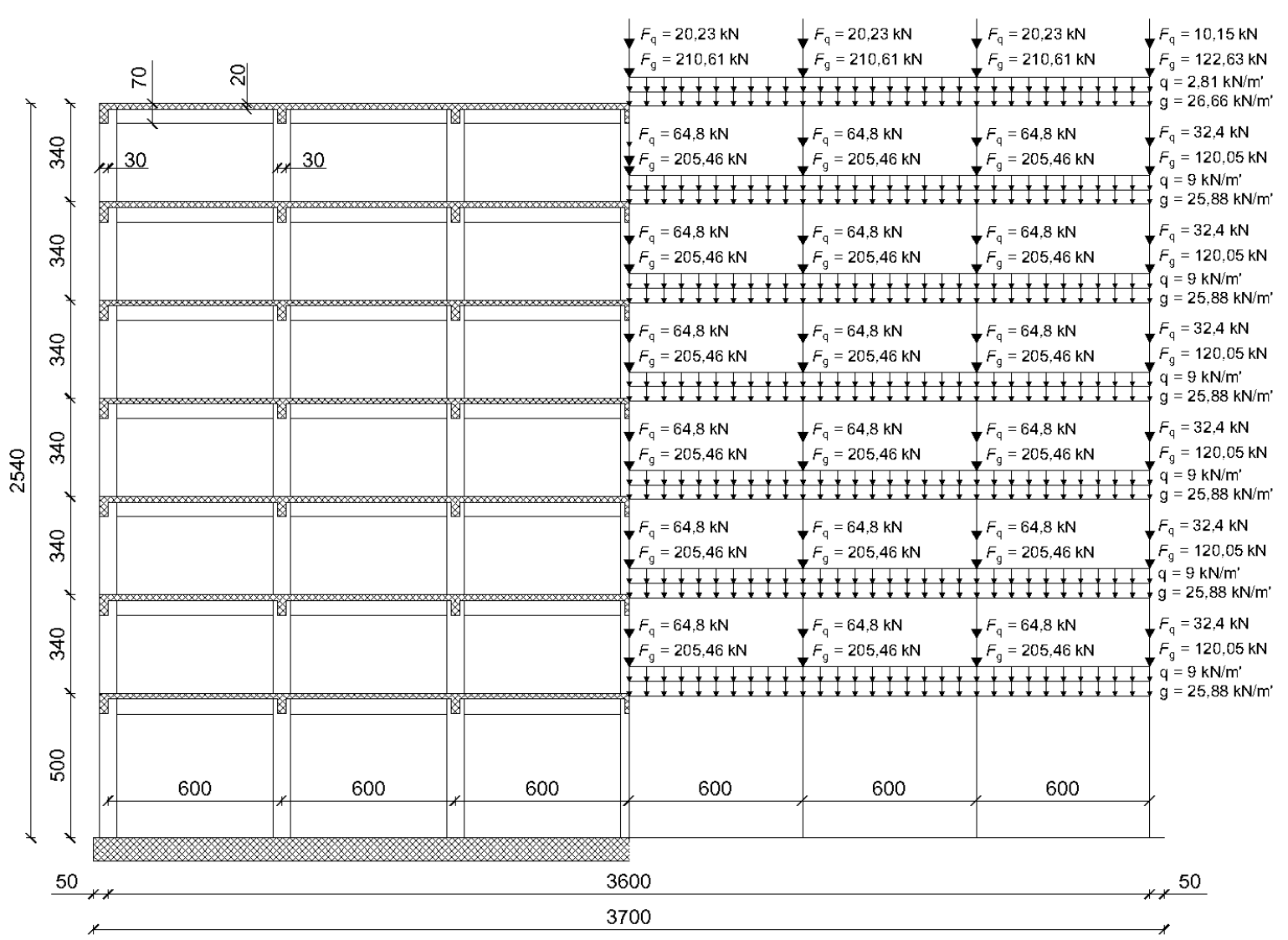

Figure 3 B6S7 structure ( $g$ is the dead load, and $q$ is the live load) [cm]

The total height of the B6S7 structure was $30.75 \mathrm{~m}$. The height of the ground floor was $5 \mathrm{~m}$, while the height of the other six stories is $3.40 \mathrm{~m}$ each. Each beam had a length of $6 \mathrm{~m}$. The structure was built using concrete class C35/45 and reinforcing steel grade B500B. Similar to the B2S6 structure, the reinforcement of the B6S7 structure was not considered because it does not affect the behavior of linearly elastic systems. All columns had a crosssection of $60 / 60 \mathrm{~cm}$ each, while all beams had a cross-section of $30 / 70 \mathrm{~cm}$ each. The slabs were $20 \mathrm{~cm}$ thick.

\subsection{Earthquake loading}

The soil-structure systems were subjected to five actual ground motion records (Table 1). The average duration of ground motion records observed was $40 \mathrm{~s}$, and all records were input in SAP2000 [11]. The main properties of ground motion records are listed in Table 1 . The predominant and mean periods were calculated using SeismoSignal [14]. Both periods indicated an approximate but useful representation of the frequency content of ground motions.

Table 1 List of actual ground motions and their basic properties

\begin{tabular}{ccccc} 
Ground motion ID & Abbreviation & $\begin{array}{c}\text { Predominant period } T_{\mathrm{P}} \\
{[\mathrm{s}]}\end{array}$ & $\begin{array}{c}\text { Mean period } T_{\mathrm{s}} \\
{[\mathrm{s}]}\end{array}$ & $\begin{array}{c}\mathbf{a}_{\mathrm{g}} \\
{\left[\mathrm{m} / \mathrm{s}^{2}\right]}\end{array}$ \\
\hline ALTADENA-1 & ALT1 & 0.36 & 0.36 & 4.39 \\
ALTADENA-2 & ALT2 & 0.14 & 0.35 & 1.76 \\
ARRAYY6-1 & ARR1 & 0.10 & 0.94 & 3.69 \\
ARRAY06-2 & ARR2 & 0.24 & 1.29 & 4.28 \\
CORRALIT-1 & COR1 & 0.30 & 0.48 & 6.18 \\
\hline \hline
\end{tabular}

Ravlić, P, Kraus, I 


\section{SOIL MODELS}

Each of the observed reinforced concrete frames was analyzed using three actual soil profiles. Two soil profiles characterized the foundation soil in Montenegro [15], and one profile described the soil stratification within the immediate vicinity of a high school in Vukovar, Croatia [16]. The three profiles are depicted in Figure 4. Soil profiles 1 and 2 were characterized by values of $v_{s, 30}$ equal to 360 and $400 \mathrm{~m} / \mathrm{s}$, respectively, and both values are placed in Category B as defined in HRN EN 1998-1 [8]. Soil profile 3, with a mean shear wave velocity of $\mathrm{v}_{\mathrm{s}, 30}$ equal to $200 \mathrm{~m} / \mathrm{s}$, belonged to the Category C according to HRN EN 1998-1 [8]. Deeper layers of soil profile 3 were not investigated, and thus, unknown to the authors. Each soil-foundation system was modeled in three ways. In the first approach, the fixed-base model was applied, representing the typical modeling approach used in engineering practice. The second approach for integrating system flexibility at the base of the structure involves using springs and dashpots. The springs and dashpots were modeled using link elements (Figure 1). The total translational stiffness in the horizontal direction $k_{x, \text { total }}$ and the total translational dashpot coefficient in the horizontal direction $c_{x, \text { total }}$ were assigned to a corner joint on the foundation (Figure 1). The total translational stiffness $k_{z, \text { total }}$ and dashpot coefficient $c_{z \text {,total }}$ in the vertical direction were equally distributed over the bottom edge of the foundation. The stiffness and damping values of the B2S6 and B6S7 models are listed in Tables 2 and 3, respectively. The third approach for modeling soil flexibility involved employing 2D thick shell elements based on the Mindlin-Reissner formulation, in which shear behavior was reflected. The shell elements were $1 \mathrm{~m}$ thick, and the soil model was discretized, which comprised approximately $0.5 / 0.5 \mathrm{~m}$ square shell elements.
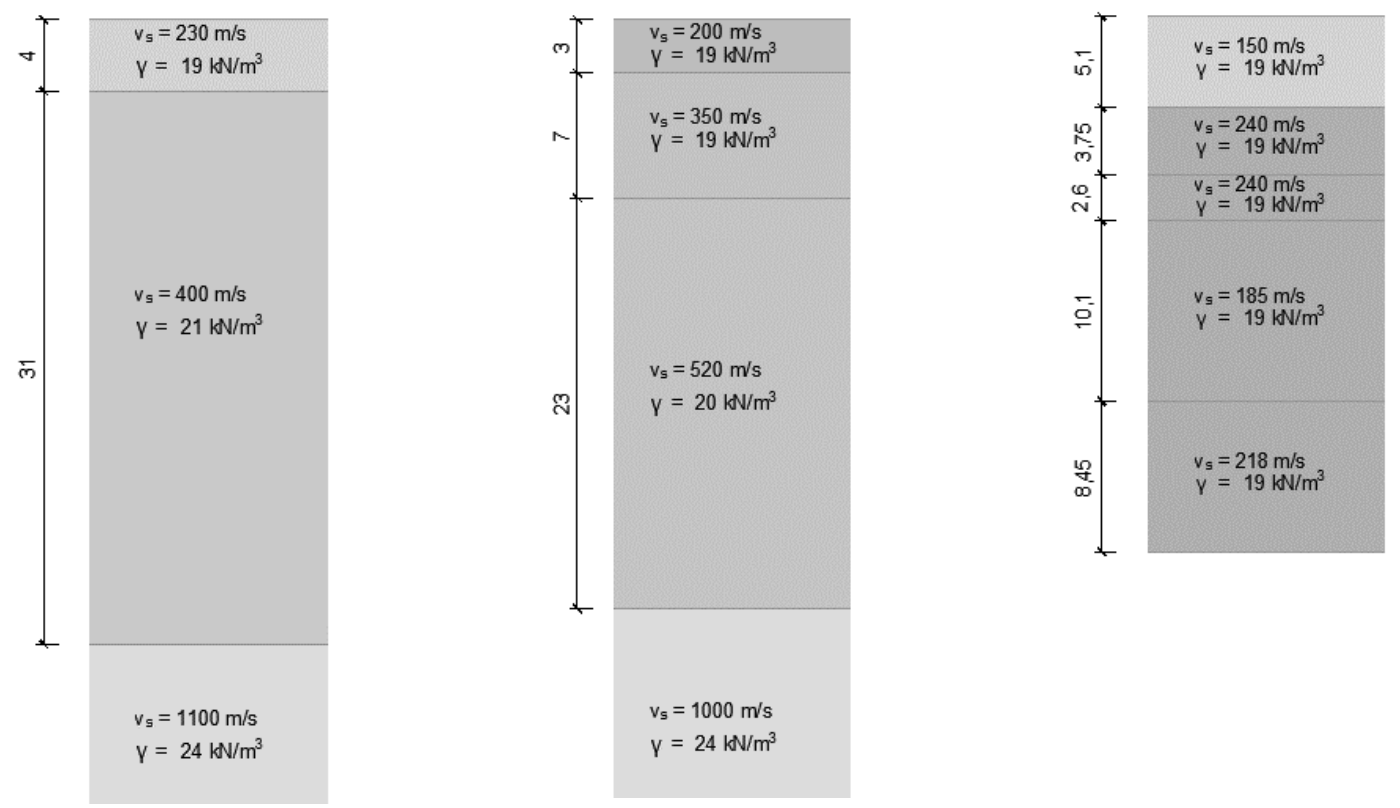

Figure 4 Soil profiles: Ulcinj, Montenegro (left), Bar, Montenegro (middle), Vukovar, Croatia (right)

Table 2 Values of stiffness and damping inputted in B2S6 model

\begin{tabular}{cccc}
\hline \multirow{2}{*}{ Sol model properties } & & Model B2S6 & Profile 3 \\
\cline { 2 - 4 } & Profile 1 & Profile 2 & 1428600 \\
$\mathrm{k}_{\mathrm{x}, \text { total }}(\mathrm{kN} / \mathrm{m})$ & 5241100 & 5667600 & 58300 \\
$\mathrm{k}_{\mathrm{z}, \mathrm{total}}(\mathrm{kN} / \mathrm{m})$ & 214000 & 231400 & 24500 \\
$\mathrm{C}_{\mathrm{x} \text { total }}(\mathrm{kNs} / \mathrm{m})$ & 48000 & 50000 & 1100 \\
$\mathrm{C}_{z, \text { total }}(\mathrm{kNs} / \mathrm{m})$ & 2175 & 2260 & \\
\hline
\end{tabular}


Table 3 Values of stiffness and damping inputted in B6S7 model

\begin{tabular}{cccc}
\hline \multirow{2}{*}{ Sol model properties } & & Model B6S7 & Profile 3 \\
\cline { 2 - 4 } & Profile 1 & Profile 2 & 2393500 \\
$\mathrm{k}_{\mathrm{x}, \text { total }}(\mathrm{kN} / \mathrm{m})$ & 8781000 & 9495500 & 45500 \\
$\mathrm{k}_{\mathrm{z}, \mathrm{total}}(\mathrm{kN} / \mathrm{m})$ & 166900 & 180500 & 57800 \\
$\mathrm{Cx}_{\mathrm{x} \text { total }}(\mathrm{kNs} / \mathrm{m})$ & 113500 & 118000 & 1110 \\
$\mathrm{C}_{\mathrm{z}, \mathrm{total}}(\mathrm{kNs} / \mathrm{m})$ & 2185 & 2270 & 1100 \\
\hline
\end{tabular}

\section{RESULTS}

The top horizontal displacements obtained from the conducted analyses are further discussed in the context of natural periods of oscillation for the soil, structure, soil-structure system, and period elongation due to SSI effects.

\subsection{Predicted fundamental periods of oscillation of structures}

The fixed-base system is denoted as FX, whereas the soil-structure system, in which the soil model consists of shell elements, is denoted as $\mathrm{S}$. The soil-structure system consisting of springs and dashpots (i.e., links) is denoted as $L$. The first three periods of oscillation for the fixed B2S6 and B6S7 models are listed in Table 4.

Table 4 Values of fundamental period of oscillation for fixed-base B2S6 and B6S7 models

\begin{tabular}{cccc}
\hline \multirow{2}{*}{ Structural model ID } & $T_{1}$ & $T_{2}$ & $T_{3}$ \\
& {$[\mathrm{~s}]$} & {$[\mathrm{s}]$} & {$[\mathrm{s}]$} \\
\hline B2S6 & 1.70 & 0.54 & 0.29 \\
B6S7 & 1.23 & 0.39 & 0.22 \\
\hline
\end{tabular}

\subsection{Predicted fundamental periods of oscillation of soil profiles}

The soil profiles investigated in this study were characterized by the average shear wave velocity in the top $30 \mathrm{~m}$. The average shear wave velocities for soil profiles 1,2 , and 3 were 360,400 , and $200 \mathrm{~m} / \mathrm{s}$, respectively. The fundamental period of oscillation for the soil was analytically estimated (Table 5) using the following expression [7]:

$$
T_{\mathrm{s}}=\frac{120}{v_{\mathrm{s}, 30}}
$$

where $v_{\mathrm{s}, 30}$ is the mean shear wave velocity in the first $30 \mathrm{~m}$ of the foundation soil.

Table 5 Oscillation periods of foundation soil

\begin{tabular}{|c|c|c|c|c|}
\hline \multirow{3}{*}{ Location } & \multirow{3}{*}{$\begin{array}{c}v_{\mathrm{s}, 30} \\
{[\mathrm{~m} / \mathrm{s}]}\end{array}$} & \multicolumn{3}{|c|}{$\begin{array}{c}T_{\mathrm{s}} \\
{[\mathrm{s}]}\end{array}$} \\
\hline & & \multirow{2}{*}{ Analytically, using equation (16) } & \multicolumn{2}{|c|}{ Numerically, using SAP2000 } \\
\hline & & & B2S6 foundation soil & B6S7 foundation soil \\
\hline Bar & 400 & 0.29 & 0.20 & 0.23 \\
\hline Ulcinj & 360 & 0.33 & 0.23 & 0.28 \\
\hline Vukovar & 200 & 0.62 & 0.45 & 0.56 \\
\hline
\end{tabular}

The fundamental oscillation period for the soil (Table 5) was numerically determined on free-field soil models comprising 2D thick shell elements using SAP2000 [11]. The lowest difference between the analytical and numerical fundamental periods of oscillation for the soil was observed for the soil model that supported the B6S7 structure (Table 5). The soil model was almost 2.5 times wider than that of the soil model developed for the B2S6 structure. The soil model for the B2S6 structure was $105 \mathrm{~m}$ wide, whereas that for the B6S7 structure was $259 \mathrm{~m}$ wide. These results confirm that wider soil models developed using shell elements produce results consistent with analytically Ravlić, P, Kraus, I 
predicted results. Moreover, such soil models simulate the infinity of the soil medium. However, the analytical expression and numerical models showed a similar increasing trend for the period of oscillation of the soil as its stiffness, i.e., the shear wave velocity decreased.

\subsection{Comparison of fundamental period of oscillation of fixed-base structure to ground motion periods}

The ratios of the fundamental periods of oscillation of the structures to ground motion periods were calculated and compared further to investigate the response of structures to ground motions. The first period of oscillation of the fixed-base model $T_{1}$ and the mean period of the ground motion record $T_{\mathrm{m}}$ are correlated as follows.

$$
K=\frac{T_{1}}{T_{m}}
$$

Figure 5 depicts the ratio of the fundamental period of oscillation of the structure to the mean period from the ground motion record. The first period of oscillation of the fixed-base model $T_{1}$ and the predominant period of the ground motion $T_{\mathrm{p}}$ are expressed as follows.

$$
\Delta=\frac{T_{1}}{T_{\mathrm{P}}}
$$

Figure 6 shows the ratio of the predominant period from the ground motion to the fundamental period of oscillation of the structure. Theoretically, the closer the ratios calculated using equations (17) and (18) are to 1, the higher the probability of resonance between the structure and the ground motion. However, from Figures 5 and 6 , it can be observed that equations (17) and (18) provide different values for the same observed ground motion. Thus, in the following subsection, the values obtained using equations (17) and (18) are correlated to the top horizontal displacements of structures to determine whether the mean period or predominant period provided better insight into possible resonance.

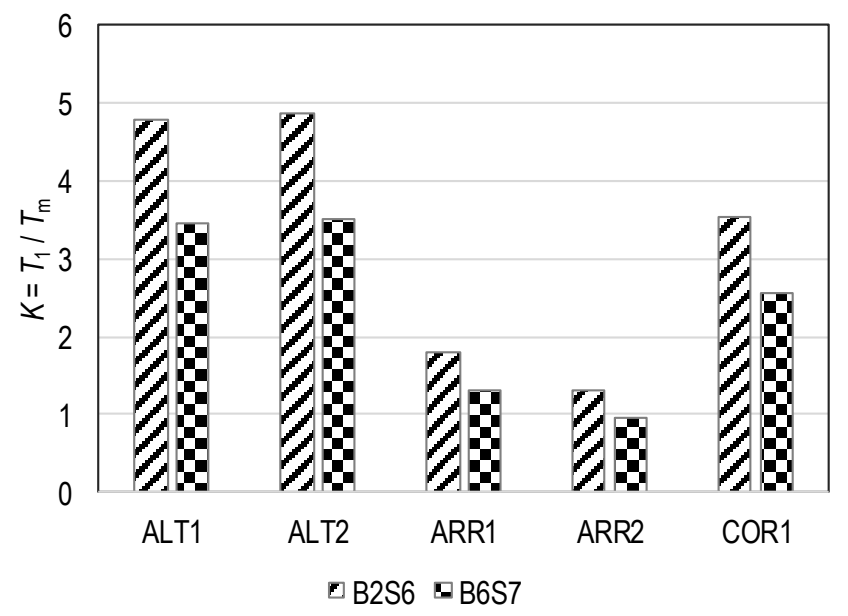

Figure 5 Ratio of first period of oscillation of fixed-base model $T_{1}$ to mean period of ground motion record $T_{\mathrm{m}}$ 


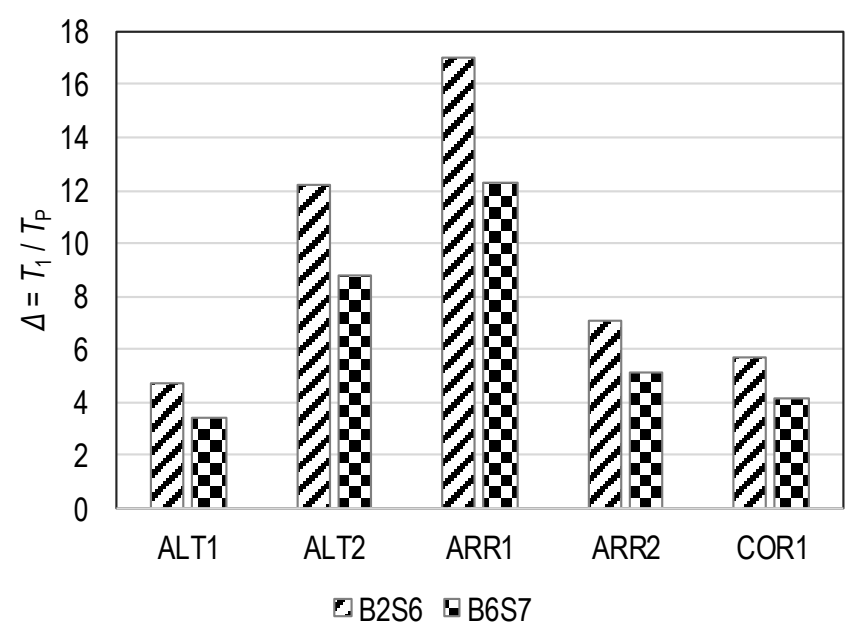

Figure 6 Ratio of first period of oscillation of fixed-base model $T_{1}$ to predominant period of ground motion record $T_{\mathrm{p}}$

\subsection{Horizontal top displacements}

By observing Figures 7 and 8 and correlating them to Figures 5 and 6 , it can be noted that the highest value of top displacements in structures produced ground motions ARR1 and ARR2. The ratios of the first period of oscillation of the fixed-base model $T_{1}$ to the mean periods of the ground motion record $T_{\mathrm{m}}$ of ground motions ARR1 and ARR2 were closest to 1. Hence, ground motions ARR1 and ARR2 did not show the highest peak acceleration value compared to other ground motions observed in this study. The same inference could be drawn for both B2S6 and B6S7 structures. Therefore, it can be concluded that the mean period of the ground motion is a good predictor of possible resonance between the structure and ground motion.

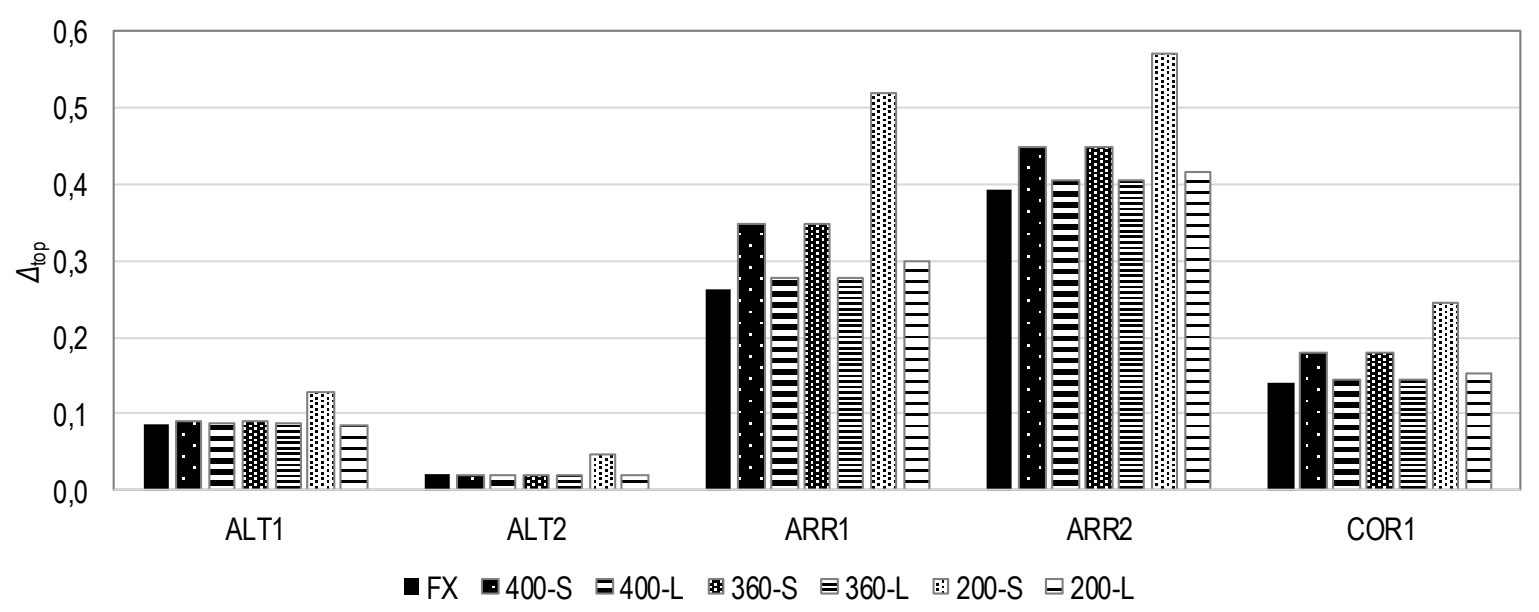

Figure 7 Horizontal top displacements of B2S6 model 


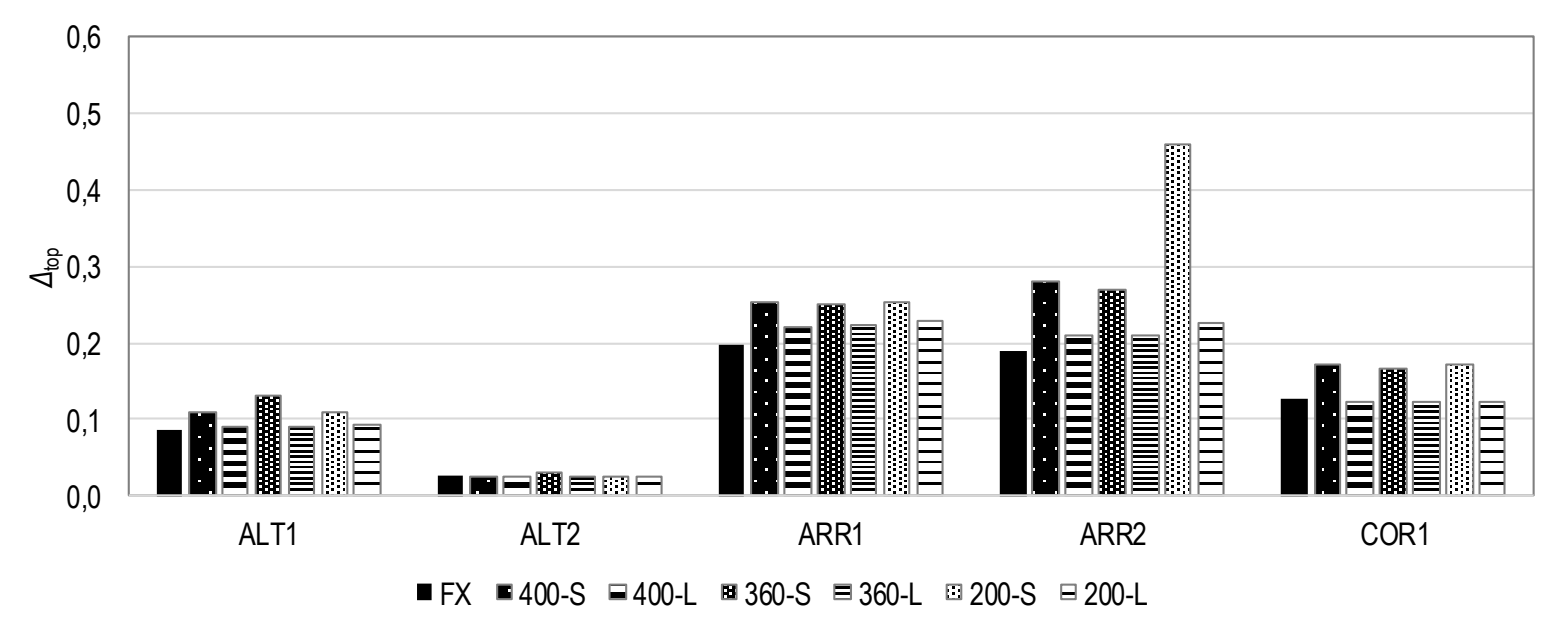

Figure 8 Horizontal top displacements of B6S7 model

This study has demonstrated that the mean period of the ground motion is a better predictor of the possible resonance than the predominant period of the ground motion. Furthermore, Figures 7 and 8 show that models with shell elements produce higher top displacements than the models with springs and dashpots (i.e., links). By analyzing Figures 7 and 8 , and in particular, by comparing the response of structures supported by soil models 400 and 360 , it was observed that the top displacement of the structure was higher when shell elements and stratified soil models were used. Soil models were established using springs and dashpots to conceal soil stratification, thus neglecting relatively softer upper soil layers (Figure 4). Hence, soil-structure systems with springs and dashpots are stiffer and yield lower horizontal top displacements than models comprising 2D finite elements and simulated stratification.

\section{CONCLUSION}

In engineering practice, numerical models of structures are typically supported using nondeformable media. However, the compliance of the underlying soil can affect the seismic response of the structure. The influence of soil compliance and stratification on the behavior of structures subjected to dynamic loads was investigated using different approaches to soil foundation modeling. Finally, the relationship between the magnitude of the top horizontal displacement of the structure and the frequency content of ground motions was established. The study was conducted for 70 soil-structure cases. Three actual soil profiles and two structures were observed and combined. In addition, the soil was modeled in three ways. The dynamic analysis was performed using SAP2000 and five records of actual ground motions.

The main findings of this study are highlighted as follows:

1) Wider soil models composed of shell elements produce the fundamental period of the foundation soil and agree with analytically predicted results. Wider soil models simulate the infinity of the medium accurately.

2) Higher top displacements in structures produce ground motions with a mean period value very close to the first period of oscillation of the fixed-base model, regardless of the peak acceleration magnitude of the ground motion.

3) The mean period of ground motion is a better predictor of possible resonance than the predominant period of ground motion.

4) The top horizontal displacement of the structure is higher when shell elements and stratified soil models are used. The soil models were made of springs and dashpots to conceal soil stratification, thus neglecting relatively softer upper soil layers. Hence, soil-structure systems with springs and dashpots are stiffer and show lower horizontal top displacements than models comprising 2D finite elements and simulated stratification.

The structures modeled in this study comprised different concrete classes. However, in linear elastic models, only the modulus of elasticity of concrete can influence the stiffness and consequently, the dynamic behavior of the 
system. In future studies, the effect of the modulus of elasticity of concrete on the behavior of the systems should be investigated.

Further tests and confirmations of the results obtained in this study should be performed using the boundary element method and specialized geotechnical software, e.g., PLAXIS.

\section{References}

[1] Özuygur, A. R.; Gunduz, A. N. 2017: Optimal Control of Structures under Earthquakes Including Soil-Structure Interaction, Journal of Earthquake Engineering, 22 (8), pp. 1317-1335. https://doi.org/10.1080/13632469.2016.1277567

[2] Badry, P. 2016: Seismic soil structure interaction analysis of piled - raft supported asymmetrical buildings, Doctoral Thesis, International Institute of Information Technology, Hyderabad 500 032, India, Available at: https://pdfs.semanticscholar.org/2cae/d53e87df0653c86c1a04ef51c74968495d20.pdf, Accessed 11 June 2019

[3] Wolf, J. P.; Deeks, A. J. 2004: Foundation Vibration Analysis. A Strength-of Materials Approach, Elsevier, Oxford, UK

[4] Maher A.; Osama A. K.; Mohamed E. 2016: Variation of seismic response of mid-rise RC buildings due to soil structure interaction effects. International Journal of Civil Engineering and Technology, 7 (1), pp. 220-240.

[5] Kraus, I.; Džakić, D. 2013: Soil-structure interaction effects on seismic behaviour of reinforced concrete frames. In: Skopje earthquake - 50 Years European Earthquake Engineering, Skopje, Macedonia

[6] Khalil, L.; Sadek, M.; Shahrour, I. 2007: Influence of the soil-structure interaction on the fundamental period of buildings. Earthquake Enginering and Structural Dynamics, 36 (15), pp. 2445-2453. https://doi.org/10.1002/eqe.738

[7] NEHRP, Consultants Joint Venture. 2012. Soil-Structure Interaction for Building Structures; NIST GCR $12-$ 917-21; NIST (National Institute of Standards and Technology), U.S. Department of Commerce: Gaithersburg, MD, USA

[8] CEN - European Committee for Standardization 2004: Eurocode 8 - Design of structures for earthquake resistance - Part 1: General rules, seismic actions and rules for buildings

[9] MacLeod, I. A. 2005: Modern structural analysis: Modelling process and guidance. Thomas Telford Publishing

[10] Tabatabaiefara, H.R.; Massumi, A. 2010: A simplified method to determine seismic responses of reinforced concrete moment resisting building frames under influence of soil-structure interaction. Soil Dynamics and Earthquake Engineering 30 (11), pp. 1259-1267. https://doi.org/10.1016/i.soildyn.2010.05.008

[11] CSI - Computers \& Structures, Inc. 2016: SAP2000, v.18.1.0. Structural analysis program, University of California, Berkley, California, USA

[12] Bisch, P. et al. 2011: Eurocode 8: Seismic Design of Buildings Worked examples, JRC Scientific and Technical Report, Available at: https://eurocodes.jrc.ec.europa.eu, Accessed 11 June 2019

[13] Čaušević, M.; Franković, T.; Mahmutović, N. 2012: Effects of stiffness reduction on seismic capacity of buildings, Građevinar, 64 (06.), pp. 463-474. https://doi.org/10.14256/JCE.668.2011

[14] Seismosoft 2016: Seismosignal 2016 - A computer program for signal processing of strong motion data, Available at: http://www.seismosoft.com Accessed 28 December 2020

[15] Talaganov, K.; Aleksovski, D.; Gadza, V. 1982: Analysis of the influence of local soil conditions upon maximum accelerations based on data from 1979 Montenegro earthquake. Proceedings of the 7th European Conference on Earthquake Engineering, Technical Chamber of Greece, Athens, Greece

[16] Faculty of Civil Engineering and Architecture Osijek 2017: Gymnasium in Vukovar, Geotechnical study, Osijek

Please cite this article as: Ravlić, P.; Kraus, I.: Influence of numerical soil model type on dynamic response of reinforced concrete frame structures, Electronic Journal of the Faculty of Civil Engineering Osijek-e-GFOS, 2020, 21, pp. 18-29, https://doi.org/10.13167/2020.21.2

Ravlić, P, Kraus, I 